\title{
Resonant peak in the density of states in normal-metal/diffusive-ferromagnet/superconductor junctions
}

\author{
T. Yokoyama, ${ }^{1}$ Y. Tanaka, ${ }^{1}$ and A. A. Golubov ${ }^{2}$ \\ ${ }^{1}$ Department of Applied Physics, Nagoya University, Nagoya, 464-8603, Japan \\ and CREST, Japan Science and Technology Corporation Nagoya, 464-8603, Japan \\ ${ }^{2}$ Faculty of Science and Technology, University of Twente, 7500 AE, Enschede, The Netherlands
}

(Received 12 April 2005; published 30 August 2005)

\begin{abstract}
The conditions for the formation of zero-energy peak in the density of states (DOS) in the normal metal/ insulator/diffusive ferromagnet/insulator/s-wave superconductor (N/I/DF/I/S) junctions are studied by solving the Usadel equations. The DOS of the diffusive-ferromagnet conductor (DF) is calculated in various regimes for different magnitudes of the resistance, Thouless energy, and the exchange field of the DF, as well as for various resistances of the insulating barriers. The conditions for the DOS peak are formulated for the cases of weak proximity effect [large resistance of the diffusive-feromagnet/superconductor (DF/S) interface] and strong proximity effect (small resistance of the DF/S interface).
\end{abstract}

DOI: 10.1103/PhysRevB.72.052512

PACS number(s): 74.50.+r

In ferromagnet/superconductor (F/S) junctions, Cooper pairs penetrating into the $\mathrm{F}$ layer from the $\mathrm{S}$ layer have a nonzero momentum due to the influence of exchange field. ${ }^{1-3}$ This results in oscillating behavior of the pair amplitude or a $\pi$-phase shift of the order parameter in the ferromagnet. A negative sign of the real part of the order parameter may occur when the thickness of the $\mathrm{F}$ layer is larger than the coherence length of the $\mathrm{F}$ layer. The occurrence of the $\pi$-phase shift makes it possible to realize the SFS $\pi$ junctions, ${ }^{1}$ as was confirmed experimentally. ${ }^{4-8}$ The order parameter oscillations also lead to nonmonotonous dependence of $T_{c}$ in SF bilayers on the F-layer thickness. ${ }^{9-13}$ Effects of resonant transmission in conductivity of SF structures were discussed in Refs. 14-16.

Another interesting consequence of the oscillations of the pair amplitude is the spatially damped oscillating behavior of the density of states (DOS) in a ferromagnet predicted theoretically ${ }^{17-20}$ in various regimes. The energy-dependent DOS calculated in the clean ${ }^{18}$ and the dirty ${ }^{21}$ limits exhibits rich structures. Experimentally DOS in F/S bilayers was measured by Kontos et al. who found a broad DOS peak around zero energy when the $\pi$-phase shift occurs. ${ }^{22}$ In diffusive-ferromagnet/superconductor (DF/S) junctions the zero-energy DOS may have a sharp peak. ${ }^{21}$ However, the conditions for the appearance of such an anomaly have not been studied systematically so far.

The purpose of the present paper is to calculate DOS in N/DF/S junctions and to formulate the conditions for the zero-energy DOS peak in two regimes corresponding to the weak proximity effect (large DF/S interface resistance) and strong proximity effect (small DF/S interface resistance). We will show that in the former case the condition is equivalent to the one of Ref. 21, while in the latter case the new condition is found. The calculation will be performed in the zero-temperature regime by varying the interface resistances as well as the resistance, the exchange field, and the Thouless energy of the DF layer.

We consider a junction consisting of normal and superconducting reservoirs connected by a quasi-one-dimensional diffusive-ferromagnet conductor (DF) with a resistance $R_{d}$ and a length $L$ much larger than the mean free path. The $\mathrm{DF} / \mathrm{N}$ interface located at $x=0$ has the resistance $R_{b}^{\prime}$, while the DF/S interface located at $x=L$ has the resistance $R_{b}$. We model infinitely narrow insulating barriers by the $\delta$ function $U(x)=H \delta(x-L)+H^{\prime} \delta(x)$. The resulting transparencies of the junctions $T_{m}$ and $T_{m}^{\prime}$ are given by $T_{m}=4 \cos ^{2} \phi /\left(4 \cos ^{2} \phi\right.$ $\left.+Z^{2}\right)$ and $T_{m}^{\prime}=4 \cos ^{2} \phi /\left(4 \cos ^{2} \phi+Z^{\prime 2}\right)$, where $Z=2 H / v_{F}$ and $Z^{\prime}=2 H^{\prime} / v_{F}$ are dimensionless constants, $\phi$ is the injection angle measured from the interface normal to the junction, and $v_{F}$ is Fermi velocity.

In the following calculation we will apply the quasiclassical Green's functions formalism. The $2 \times 2$ retarded Green's functions in $\mathrm{N}, \mathrm{DF}$, and $\mathrm{S}$ are denoted by $\hat{R}_{0}(x)$, $\hat{R}_{1}(x)$, and $\hat{R}_{2}(x)$ respectively. $\hat{R}_{0}(x)$ and $\hat{R}_{2}(x)$ are expressed by $\hat{R}_{0}(x)=\hat{\tau}_{3}$ and $\hat{R}_{2}(x)=\left(g \hat{\tau}_{3}+f \hat{\tau}_{2}\right) \quad$ respectively, with $g=\varepsilon / \sqrt{\varepsilon^{2}-\Delta^{2}}$ and $f=\Delta / \sqrt{\Delta^{2}-\varepsilon^{2}}$, where $\hat{\tau}_{2}$ and $\hat{\tau}_{3}$ are the Pauli matrices, and $\Delta$ and $\varepsilon$ denote the energy gap and the quasiparticle energy measured from the Fermi energy, respectively. It is convenient to use the standard $\theta$ parametrization when function $\hat{R}_{1}(x)$ is expressed as $\hat{R}_{1}(x)$ $=\hat{\tau}_{3} \cos \theta(x)+\hat{\tau}_{2} \sin \theta(x)$. The parameter $\theta(x)$ is a measure of the proximity effect in DF. The spatial dependence of $\theta(x)$ in $\mathrm{DF}$ is determined by the static Usadel equation ${ }^{23}$

$$
D \frac{\partial^{2}}{\partial x^{2}} \theta(x)+2 i(\varepsilon-(+) h) \sin [\theta(x)]=0
$$

for majority (minority) spin with the diffusion constant $D$ and the exchange field $h$ in DF. Note that we assume a weak ferromagnet and neglect the difference of the Fermi velocities of the majority and minority spin subbands.

Further we shall apply the Nazarov's boundary condition $^{24,25}$ for $\theta(x)$ at both interfaces. At the DF/N interface it has the following form: 


$$
\begin{gathered}
\left.\frac{L}{R_{d}} \frac{\partial \theta(x)}{\partial x}\right|_{x=0_{+}}=\frac{\langle F\rangle^{\prime}}{R_{b}^{\prime}}, \\
F=\frac{2 T_{m}^{\prime} \sin \theta\left(0_{+}\right)}{\left(2-T_{m}^{\prime}\right)+T_{m}^{\prime} \cos \theta\left(0_{+}\right)},
\end{gathered}
$$

and it has a similar form at the DF/S interface. This boundary condition is based on the Zaitsev's boundary condition ${ }^{26}$ with isotropic limit and generalizes the Kupriyanov-Lukichev boundary condition. ${ }^{27}$

The average over the various angles of injected particles at the interface is defined as

$$
\langle B(\phi)\rangle^{\prime}=\frac{\int_{-\pi / 2}^{\pi / 2} d \phi \cos \phi B(\phi)}{\int_{-\pi / 2}^{\pi / 2} d \phi T^{\prime}(\phi) \cos \phi},
$$

with $B(\phi)=B$ and $T^{\prime}(\phi)=T_{m}^{\prime}$. The resistance of the interface $R_{b}^{(\prime)}$ is given by

$$
R_{b}^{(\prime)}=R_{0}^{(\prime)} \frac{2}{\int_{-\pi / 2}^{\pi / 2} d \phi T^{(\prime)}(\phi) \cos \phi} .
$$

Here, for example, $R_{b}^{(\prime)}$ denotes $R_{b}$ or $R_{b}^{\prime}$, and $R_{0}^{(\prime)}$ is Sharvin resistance, which in three-dimensional case is given by $R_{0}^{(\prime)-1}=e^{2} k_{F}^{2} S_{c}^{(\prime)} /\left(4 \pi^{2}\right)$, where $k_{F}$ is the Fermi wave vector and $S_{c}^{(\prime)}$ is the constriction area.

In the following, we will study the local DOS $N$ in the DF layer which is given by

$$
N / N_{0}=\frac{1}{2} \sum_{\uparrow, \downarrow} \operatorname{Re} \cos \theta(x),
$$

where $N_{0}$ denotes the DOS in the normal state. The DOS will be calculated by numerical solution of the Usadel equations with the boundary conditions given above.

Below we will concentrate on the DOS at $x=0$ (N/DF interface) in the regime of large resistance of the N/DF interface, $R_{d} / R_{b}^{\prime} \ll 1$, and will also fix the barrier transparency parameters $Z=3, Z^{\prime}=3$.

In order to study the condition for the appearance of the zero-energy DOS peak, we plot the normalized zero-energy DOS at $x=0$ as a function of $E_{T h}=D / L^{2}$. Figure 1 shows the DOS for $R_{d} / R_{b}^{\prime}=0.1$ and various $h / \Delta$. In Fig. 1(a) the zeroenergy peak appears at $E_{T h} \sim 2 h R_{b} / R_{d}$, while in Fig. 1(b) and 1(c) the peak appears at $E_{T h} \sim h$. Thus we can conclude that the condition for the DOS peak for large $R_{d} / R_{b}$ is essentially different from the one for small $R_{d} / R_{b}$.

Figure 2 shows the DOS as a function of $\varepsilon$ for the parameters corresponding to the peaks in Fig. 1 for various $h / \Delta$. In all these cases the DOS peak appears around zero energy. For small $h / \Delta$ the DOS peak is narrow but it becomes broader with the increase of $h / \Delta$. It's important to note that this peak does not always require the sign change of pair amplitude. This is also clear from the fact that the peak occurs for large Thouless energy (short DF) when there is no sign change.
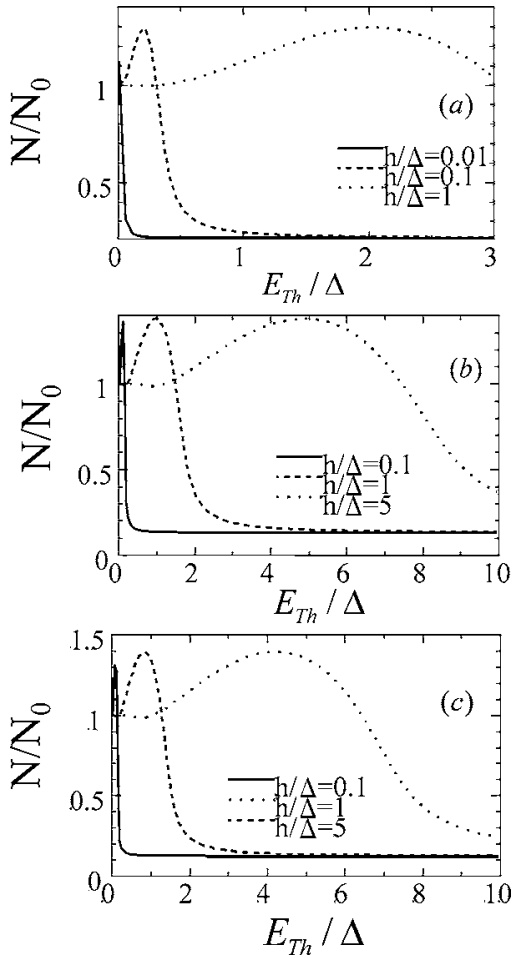

FIG. 1. Normalized zero energy DOS as a function of $E_{T h}$ for large resistance of the N/DF interface $R_{d} / R_{b}^{\prime}=0.1$ and various $h / \Delta$ with resistance ratios at the $\mathrm{DF} / \mathrm{S}$ interface (a) $R_{d} / R_{b}=1$, (b) $R_{d} / R_{b}=5$, and (c) $R_{d} / R_{b}=10$.
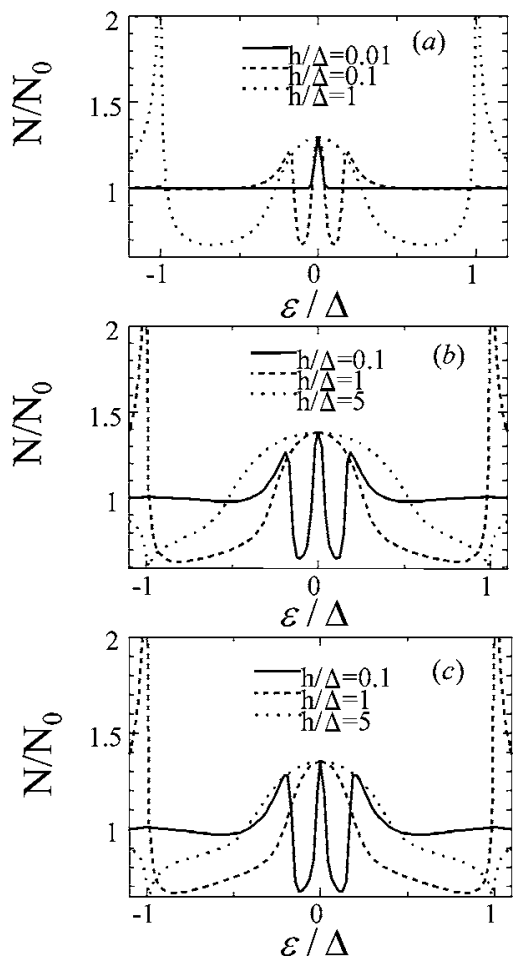

FIG. 2. Normalized DOS as a function of $\varepsilon$ for $R_{d} / R_{b}^{\prime}=0.1$ and various $h / \Delta$ with (a) $R_{d} / R_{b}=1$ and $E_{T h}=2 h R_{b} / R_{d}=2 h$, (b) $R_{d} / R_{b}$ $=5$ and $E_{T h}=h$, and (c) $R_{d} / R_{b}=10$ and $E_{T h}=h$. 

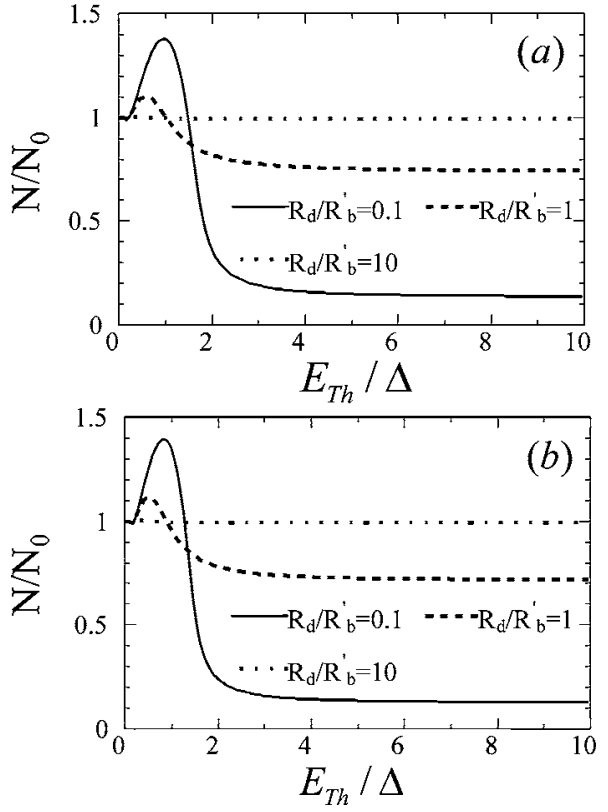

FIG. 3. Normalized DOS at zero energy as a function of $E_{T h}$ for $h / \Delta=1$ and various $R_{d} / R_{b}^{\prime}$ with (a) $R_{d} / R_{b}=5$ and (b) $R_{d} / R_{b}=10$.

For other sets of parameters, the DOS peak is smeared as they break the condition $E_{T h} \sim 2 h R_{b} / R_{d}$ or $E_{T h} \sim h$.

Let us first discuss the case of strong proximity effect in detail. Figure 3 shows the zero-energy DOS at $x=0$ as a function of $E_{T h}$ for $h / \Delta=1$ and various $R_{d} / R_{b}^{\prime}$ with (a) $R_{d} / R_{b}=5$ and (b) $R_{d} / R_{b}=10$. In this case the peak at $E_{\mathrm{Th}}$ $\sim h$ is suppressed with increasing $R_{d} / R_{b}^{\prime}$. Therefore this condition is valid for small $R_{d} / R_{b}^{\prime}$.

Figure 4 shows the spatial dependence of $\operatorname{Im} \theta$ for majority spin for $R_{d} / R_{b}^{\prime}=0.1, E_{T h} / \Delta=1$, and various $h / \Delta$ with (a) $R_{d} / R_{b}=5$ and (b) $R_{d} / R_{b}=10$. For the appearance of the DOS peak, large value of $\operatorname{Im} \theta$ is needed because the normalized DOS is given by $\operatorname{Re} \cos (\theta)=\cos [\operatorname{Re}(\theta)] \cosh [\operatorname{Im}(\theta)]$. As seen from Fig. 4, the magnitude of $\operatorname{Im} \theta$ increases with the increase of the distance from the DF/S interface and achieves a maximum when $E_{T h}=h$.

Note that the zero-energy DOS at $x=0$ does not depend on $E_{T h}$ if the condition $E_{T h}=h$ holds. To explain that, let's write Eqs. (1) and (2) at $\varepsilon=0$ :

$$
\begin{gathered}
\frac{\partial^{2}}{\partial y^{2}} \theta(y)-(+) 2 i \sin [\theta(y)]=0, \\
\left.\frac{1}{R_{d}} \frac{\partial \theta(y)}{\partial y}\right|_{y=0_{+}}=\frac{\langle F\rangle^{\prime}}{R_{b}^{\prime}},
\end{gathered}
$$

where $y \equiv x / \sqrt{D / h}$. Since for $E_{T h}=h$ we have $D / h$ $\equiv E_{T h} L^{2} / h=L^{2}$, the above equations don't contain $E_{T h}$ as a parameter. Similar arguments can be applied to another boundary condition at DF/S interface. This proves the above statement about independence of the zero-energy DOS at $x=0$ on $E_{T h}$.

Now let us discuss the weak proximity effect and derive the condition $R_{d} / R_{b} \sim 2 h / E_{T h}$, following Ref. 21 . When spatial variation of $\theta$ is small, i.e., $L \ll \sqrt{D /|\varepsilon \mp h|}$ (for the
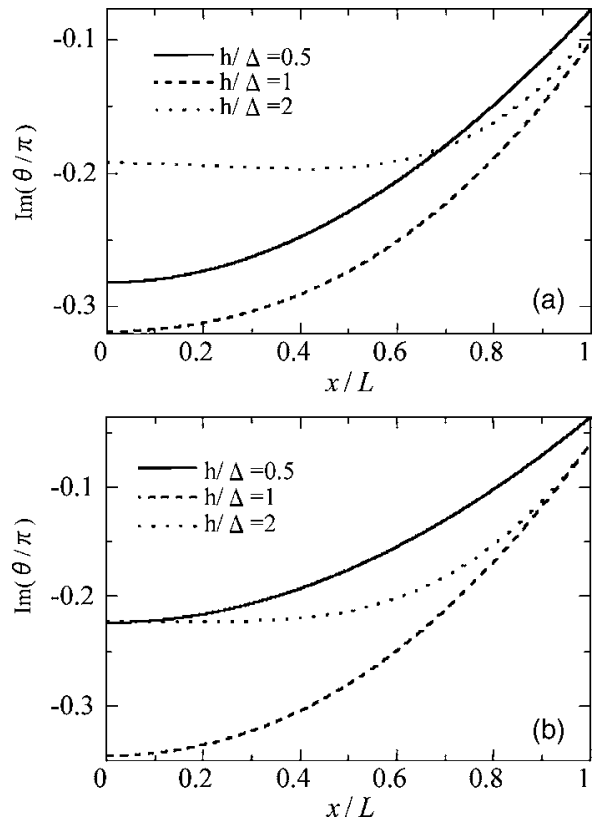

FIG. 4. Spatial dependence of $\operatorname{Im} \theta$ for majority spin for $R_{d} / R_{b}^{\prime}=0.1, E_{T h} / \Delta=1$ and various $h / \Delta$ with (a) $R_{d} / R_{b}=5$ and (b) $R_{d} / R_{b}=10$. The DF/N interface and the DF/S interface are located at $x=0$ and $x=L$ respectively.

spin-up or spin-down subband respectively) and both $R_{d} / R_{b}$ and $R_{d} / R_{b}^{\prime}$ are small (weak proximity effect), $\theta$ can be expanded as $\theta=\theta_{0}+\theta_{1} x+\theta_{2} x^{2}$ where $\theta_{1}, \theta_{2} \ll \theta_{0}$. Note that the derivatives of $\theta$ are proportional to these quantities at the interfaces [see Eq. (2) and Ref. 25].

In this case the solution of the Usadel equation in the spin-up subband satisfying boundary conditions has the form:

$$
\cos \theta_{0 \uparrow}=\frac{\frac{R_{d}}{R_{b}^{\prime}}+\frac{R_{d}}{R_{b}} g-\frac{2 i(\varepsilon-h)}{E_{T h}}}{\sqrt{\left(\frac{R_{d}}{R_{b}} f\right)^{2}+\left(\frac{R_{d}}{R_{b}^{\prime}}+\frac{R_{d}}{R_{b}} g-\frac{2 i(\varepsilon-h)}{E_{T h}}\right)^{2}}} .
$$

For $R_{d} / R_{b}^{\prime}=0$ and $\varepsilon=0$, the DOS has the form

$$
\cos \theta_{0 \uparrow}=\frac{\frac{2 i h}{E_{T h}}}{\sqrt{\left(\frac{R_{d}}{R_{b}}\right)^{2}-\left(\frac{2 h}{E_{T h}}\right)^{2}}},
$$

which provides the resonant condition $R_{d} / R_{b} \sim 2 h / E_{T h}$. Similar result follows for the spin-down subband by replacing $h$ by $-h$.

Another resonant condition for the strong proximity effect, $E_{T h} \sim h$, is equivalent to the condition $L \sim \sqrt{D / h}$. Thus, zero-energy DOS peak appears when the proximity effect is strong and the length of ferromagnet is of the order of the coherence length in a ferromagnet $\xi_{F}=\sqrt{D / h}$.

Let us discuss the physical meaning of two conditions. In DN/S junctions there is a minigap $E_{g}$, where $E_{g} \sim E_{T h} R_{d} / R_{b}$ 
for weak proximity effect, or $E_{g} \sim E_{T h}$ for strong proximity effect. ${ }^{28}$ In DF/S junctions this minigap is shifted by $h$, then the DOS peak appears when $h \sim E_{g}$.

Note that in the calculations we have fixed $Z=Z^{\prime}=3$, but the specific parameter choice does not change the results qualitatively.

In summary, we have studied the conditions for the appearance of the DOS peak in diffusive ferromagnet, in normal metal/diffusive ferromagnet/s-wave superconductor junctions. We have discussed two regimes of weak and strong proximity effect depending on the ratio $R_{d} / R_{b}$. The results in the regime of weak proximity effect are essentially the same as found in Ref. 21. However, in the regime of strong proximity effect the results are qualitatively different. Let us summarize the two conditions:

(1) When the proximity effect is weak $\left(R_{d} / R_{b} \ll 1\right)$, the condition for the DOS peak is $R_{d} / R_{b} \sim 2 h / E_{T h}$.

(2) When the proximity effect is strong $\left(R_{d} / R_{b} \gg 1\right)$, the DOS peak appears when $E_{T h} \sim h$, i.e. when the length of ferromagnet is of the order of the coherence length $\sqrt{D / h}$.

Note that the above two conditions cross over into each other when $R_{d} / R_{b} \sim 2$. Since the DOS is a fundamental quantity affecting various physical properties, our results may have many applications, e.g., for the conductance of N/DF/S structures.

The authors appreciate useful and fruitful discussions with J. Inoue, M. Yu. Kupriyanov, A. Kadigrobov, Yu. Nazarov, and H. Itoh. This work was supported by a Grant-inAid for the 21st Century COE "Frontiers of Computational Science" and by INTAS Grant No. 01-0809.
${ }^{1}$ A. I. Buzdin, L. N. Bulaevskii, and S. V. Panyukov, Pis'ma Zh. Eksp. Teor. Fiz. 35, 147 (1982) [JETP Lett. 35, 178 (1982)].

${ }^{2}$ A. I. Buzdin and M. Yu. Kupriyanov, Pis'ma Zh. Eksp. Teor. Fiz. 53, 308 (1991) [JETP Lett. 53, 321 (1991)].

${ }^{3}$ E. A. Demler, G. B. Arnold, and M. R. Beasley, Phys. Rev. B 55, 15174 (1997).

${ }^{4}$ V. V. Ryazanov et al., Phys. Rev. Lett. 86, 2427 (2001); S. M. Frolov et al., Phys. Rev. B 70, 144505 (2004).

${ }^{5}$ T. Kontos et al., Phys. Rev. Lett. 89, 137007 (2002).

${ }^{6}$ Y. Blum et al., Phys. Rev. Lett. 89, 187004 (2002).

${ }^{7}$ H. Sellier et al., Phys. Rev. B 68, 054531 (2003).

${ }^{8}$ A. Bauer et al., Phys. Rev. Lett. 92, 217001 (2004).

${ }^{9}$ Z. Radovic et al., Phys. Rev. B 44, 759 (1991).

${ }^{10}$ L. R. Tagirov, Phys. Rev. Lett. 83, 2058 (1999).

${ }^{11}$ Ya. V. Fominov, N. M. Chtchelkatchev, and A. A. Golubov, Phys. Rev. B 66, 014507 (2002).

${ }^{12}$ A. Rusanov et al., Physica C 369, 300 (2002).

${ }^{13}$ V. V. Ryazanov et al., JETP Lett. 77, 39 (2003).

${ }^{14}$ A. Kadigrobov et al., Phys. Rev. B 60, 14593 (1999).

${ }^{15}$ M. Leadbeater et al., Phys. Rev. B 59, 12264 (1999).

${ }^{16}$ R. Seviour, C. J. Lambert, and A. F. Volkov, Phys. Rev. B 59,
6031 (1999).

${ }^{17}$ A. Buzdin, Phys. Rev. B 62, 11377 (2000).

${ }^{18}$ M. Zareyan, W. Belzig, and Yu. V. Nazarov, Phys. Rev. Lett. 86, 308 (2001); Phys. Rev. B 65, 184505 (2002).

${ }^{19}$ I. Baladie and A. Buzdin, Phys. Rev. B 64, 224514 (2001).

${ }^{20}$ F. S. Bergeret, A. F. Volkov, and K. B. Efetov, Phys. Rev. B 65 , 134505 (2002).

${ }^{21}$ A. A. Golubov, M. Yu. Kupriyanov, and Ya. V. Fominov, JETP Lett. 75, 223 (2002).

${ }^{22}$ T. Kontos et al., Phys. Rev. Lett. 86, 304 (2001); 93, 137001 (2004).

${ }^{23}$ K. D. Usadel, Phys. Rev. Lett. 25, 507 (1970).

${ }^{24}$ Yu. V. Nazarov, Superlattices Microstruct. 25, 1221 (1999).

${ }^{25}$ Y. Tanaka, A. A. Golubov, and S. Kashiwaya, Phys. Rev. B 68, 054513 (2003).

${ }^{26}$ A. V. Zaitsev, Sov. Phys. JETP 59, 1163 (1984).

${ }^{27}$ M. Yu. Kupriyanov and V. F. Lukichev, Zh. Eksp. Teor. Fiz. 94, 139 (1988) [Sov. Phys. JETP 67, (1988) 1163].

${ }^{28}$ A. A. Golubov, F. K. Wilhelm, and A. D. Zaikin, Phys. Rev. B 55, 1123 (1997). 